\title{
MATTHÄUS MERIAN EL JOVEN, PINTOR EN EL TALLER DE VAN DYCK: A PROPÓSITO DE UN LIENZO CON VERTUMNO Y POMONA IDENTIFICADO
}

POR

\author{
JAHEL SANZSALAZAR
}

\begin{abstract}
El lienzo con Vertumno y Pomona, con la firma "MMerian pinxit", es objeto de aportación al catálogo de Matthäus Merian el Joven, y motivo para recordar a este pintor, cuya presencia junto a Van Dyck en Londres en 1639 no ha tenido eco en los estudios dedicados al flamenco. Del análisis del estilo y las fuentes de inspiración se desprenden coincidencias con otros dos pintores muy vandyckianos, Thomas Willeboirts Bosschaert y Peeter Thijs, lo que abre un interesante circuito de transmisión de influencias e interconexiones a la sombra de Van Dyck, donde se forjaron sus estilos. Así, en busca de mayor luz sobre el tan desconocido taller inglés del pintor, se recrean las circunstancias que condicionarían el ingreso del joven Merian en su estudio, apuntando un probable encuentro con Peeter Thijs.
\end{abstract}

Palabras clave: Merian; Van Dyck; Taller de Van Dyck; Londres; Thijs; Willeboirts Bosschaert; Pintura flamenca.

The painting of Vertumnus and Pomona, signed "MMerian pinxit," is a new contribution to Matthäus Merian the Younger's oeuvre. This discovery offers an occasion to call attention to the artist, whose presence from 1639 in Anton van Dyck's London studio has been overlooked. Analyses of style and sources of inspiration suggest coincidences with two other very vandyckian painters, Thomas Willeboirts Bosschaert and Peeter Thijs, which disclose a most interesting circuit of influences and interconnections in the shadow of Van Dyck, under whose impact their styles took shape. Thus, in search of more light on the master's relatively unknown London studio, the circumstances in which the young Merian came into contact with Van Dyck are reconstructed, while suggesting a virtual meeting with Peeter Thijs.

Key words: Merian; Van Dyck; Van Dyck's studio; London; Thijs; Willeboirts Bosschaert; Flemish Painting.

Fue durante nuestra investigación sobre la obra de Van Dyck en España, cuando llegó a nuestro conocimiento una pintura muy vandyckiana, lienzo de grandes dimensiones y estimable calidad con la historia de Vertumno y Pomona (fig. 1) ${ }^{1}$. Al tiempo que buscábamos su autor en el entorno más

${ }^{1}$ Lienzo, $186 \times 130 \mathrm{~cm}$, Barcelona, colección particular. 
conocido de Van Dyck, fuimos advertidos de la presencia de una firma en el extremo inferior derecho del lienzo en curso de limpieza ${ }^{2}$. La lectura de "MMerian pinxit" (fig. 2) da motivo de aportación al catálogo de Matthäus Merian el Joven (Basilea, 1621-1687), pintor al que se ha prestado poca atención; no obstante objeto de una monografía publicada en 2002 y de otros pocos trabajos ${ }^{3}$.

Matthäus Merian el Joven es nombrado fundamentalmente por sus retablos en las iglesias alemanas y por su trabajo como retratista al servicio del mariscal de Suecia, Carl Gustav Wrangler. Por su actividad en las cortes de Fráncfort, Nüremberg y Bamberg fue considerado como "el mejor pintor de Alemania", en palabras del embajador francés, en $1663^{4}$. Pero, pese a este elogioso comentario y a la reputación que obtuvo en su época ${ }^{5}$, la obra de Matthäus Merian el Joven permanece en la penumbra y menos estudiada que la de su padre, el grabador Matthäus Merian el Viejo, célebre por su Theatrum Europeaum y sus Topographia Germaniae ${ }^{6}$ y la de su hermana, Maria Sibyilla Merian, reconocida entomóloga y pintora especializada en flores e insectos?

El estudio de Vertumno y Pomona permite conocer mejor la obra de Matthäus Merian el Joven, pero también es motivo para subrayar la relación del pintor con Van Dyck. Escasamente señalada en los estudios dedicados a éste ${ }^{8}$, la presencia de Merian junto a Van Dyck en Londres es un hecho olvidado. Según consta en su Autobiografía, después de viajar con su primer maestro, el célebre Joachim van Sandrart, Merian estuvo en el taller de Van Dyck desde el otoño de 1639

\footnotetext{
${ }^{2}$ La firma fue hallada por Inmaculada Alonso en el extremo inferior derecho del lienzo. Procedió a una limpieza de la superficie, levantando barnices oxidados y burdos repintes, revelándose arrepentimientos en la mano derecha del amorcillo en primer término. Nuestro agradecimiento a Inmaculada Alonso por su comunicación; a Matthias Weniger, que nos facilitó antiguas publicaciones alemanas; y a James Lattimer, que nos ayudó a comprender mejor los textos en alemán.

${ }^{3}$ Nieden, D., Matthäus Merian der Jüngere (1621-1687), Göttingen, Cuvillier Verlag, 2002. Previamente sobre el pintor: WaCKernagel, R., ed., "Selbstbiographie des jüngeren Matthäus Merian“, Basler Jahrbuch, 1895, pp. 227-244; Konow, H., "Eine Zeichnungssammlung aus den Besitz Matthäus Merians des Jüngeren. Ein Beitrag zur Geschichte des Berliner Kupferstichkabinetts“, Berliner Museen, 1940, pp. 58-63; MAKes, F., Investigation, Restoration and Conservation of Matthaeus Merian Portraits, Göteborg Studies in Conservation, 3, Goteborg, 1996.

${ }^{4}$ Cit. Wüthrich, L., "Matthäus Merian (ii)", in The Dictionary of Art, J. Turner (ed.), p. 152.

5 Vid. Peltzer, A. R., Joachim von Sandrarts Academie der Bau, Bild und Mahlerey Künste von 1675, Múnich, 1925 , p. 200

${ }^{6}$ Matthäus Merian el Viejo (1593-1650), ilustró también la biblia de Lutero, la llamada "Biblia-Merian"; grabó y produjo mapas topográficos, la Archontologica cosmica (1638); [véase BINGEL, H., Das Theatrum Europaeum, SÄNDIG, M. (1909), 1969; Wüthrich, L. H., Die Handzeichnungen von Matthäus Merian d. Ae. Unter Berücksichtigung der fraglichen Zuschreibungen sowie der Gemälde, nebst einem Exkurs ubre die Werke der Monogrammisten MM um 1610, Basel, 1963; Idem, Das druckgraphische Werk von Matthäus Merian d.Ä., 4 vols., Basel, 1966-1995; Idem, Register zu Merinas "Topographia Germaniae, Kassel-Basel, 1967; Matthäus Merinas der Altere, Cat. Exp. Frankfurt am MainBasel, 1993-94; Cuadriello, J., "Una biblia para el Nuevo Mundo: la Conquista de México y los emblemas políglotas de Matthaeus Merian”, en López PozA, S. (ed.), Florilegio de estudios de Emblemática. A Florilegium of Studies on Emblematics. Actas del VI Congreso Internacional de Emblemática de The Society for Emblem Studies. Proceedings of the 6th International Conference of The Society for Emblem Studies, A Coruña, 2002, Ferrol, 2004, pp. 33-48].

7 RÜCKer, E., Maria Sibylla Merian, 1647-1717. Ihr Werken in Deutschland und Holland, Nachbarn, 1980; Maria Sibylla Merian: Leben und Werk, 1647-1717, Cat. Exp. Basel, Phister Burkhalter, M., 1980; Kerner, C., Seidenraupe, Dschungelblüte: die Lebensgeschichte der Maria Sibylla Merian, Weinheim, 1989; DAvIS, N. Z., Women on the margins: three seventeenth century lives, Cambridge, 1995; KAISER, H., Maria Sibylla Merian: eine Biographie, Zurich, 1997, Dusseldorf, 2001; Keppler, U., Die Falterfrau: Maria Sibylla Merian, Múnich, 1999; KüHn, D., Frau Merian! Eine Lebensgeschichte, Fráncfort, 2002.

${ }^{8}$ Michiels incluyó a Mathaüs Merian el Joven entre los pintores tocados por la estela de Van Dyck [MichIELS, A., Van Dyck et ses élèves, París, 1882, p. 563]; pero la noticia de su presencia en Londres en 1639, recogida por el manuscrito anónimo publicado por LARSEN, E. ["La Vie, les ouvrages et les élèves de Van Dyck. Manuscrit inédit du Louvre par un auteur anonyme", Academie Royale de Belgique. Mémoires de la classe des Beaux Arts, 14, 2, 1975, p. 80, 121] y por Cust, L. [Anthony van Dyck. An Historical Study of his Life and Works, Londres, 1900, p. 151], no ha tenido eco en las publicaciones posteriores sobre Van Dyck.
} 


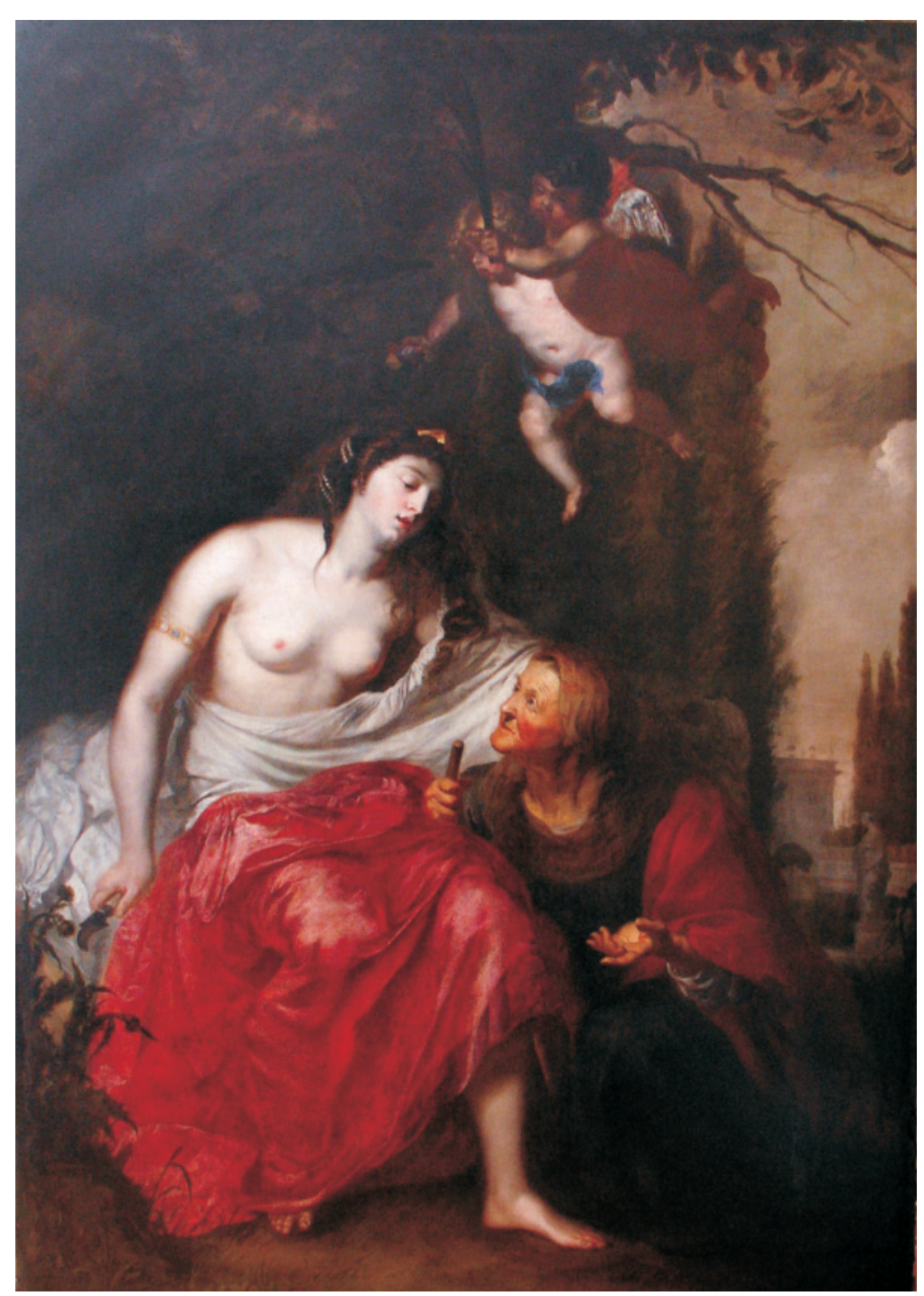

Fig. 1. Matthäus Merian el Joven. Vertumno y Pomona. Barcelona, colección particular.

hasta la muerte del flamenco9. Van Dyck le "permitió pintar a su lado por algún tiempo" y lo tuvo en "alta estima". Nos cuenta que no aceptó las 50 libras anuales que Van Dyck le ofreció en pago por considerarse ya suficientemente honrado y que entró allí por recomendación de Michel Le Blon ${ }^{10}$, personaje en el que más adelante nos detendremos.

9 "Nachdem in Engeland die Kriegstrublen untern König Carle den Ersten ansiengen, so begunte man die junge Leuthe zu pressen. Ulso begabe ich mich a. ${ }^{\circ} 1641$, da eben auch Van Dyck. Tottes verblichen ware, naher Rye über See nach Diebe, Ruan und Parys" [Wackernagel, op. cit. 1895, p. 231], noticia que confirma Sandrart [Sandrart, op. cit. 1675, in Peltzer, op. cit. 1925, p. 200].

10 “(...) alda in London durch Herrn Michael Le Blon, königl. Schedischen Agenten, Recommandation ich zu Herrn Anthoni van Dyck comen bin, daâ er bei ihm mahlen mir vergönte, welche Zeit ich dann gar wohl in Acht nahme. Er 


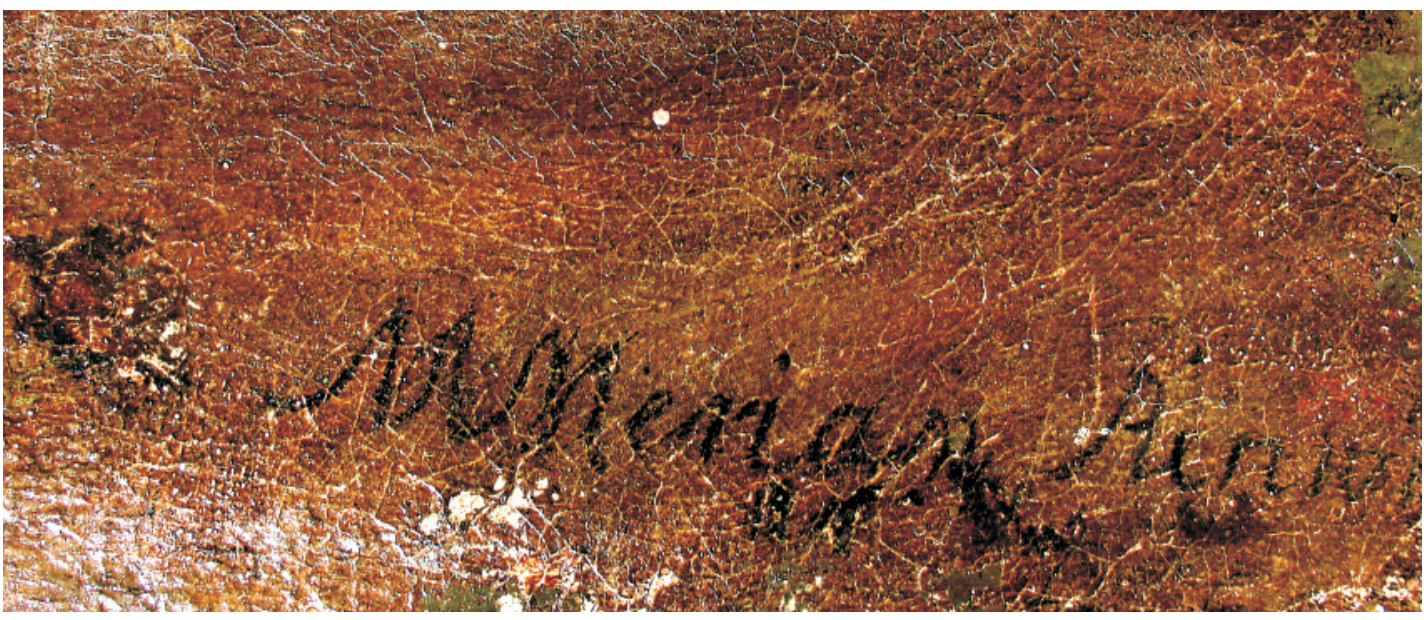

Fig. 2. Detalle de la firma.

Matthaüs Merian el Joven revela en esta pintura el buen hacer de sus mejores obras: los retratos de Zacharias Stenglin del Historisches Museum de Fráncfort, el de Karl Gustav Wrangler en Estocolmo y el de Matthäus Merian el Viejo en el Kunsthistorisches Museum de Viena, así como los retablos de la Catedral de Bamberg y las dos versiones de Artemisa, en Missouri y Dessau ${ }^{11}$. Tal calidad, sin embargo, no está en todos los retratos de su producción; algunos son producto de numerosos encargos y sin duda ejecutados en serie.

Se trata aquí de una de las pocas pinturas con asunto mitológico que hasta la fecha se conocen de su mano, por lo que permite observar su manera de expresarse en esta otra modalidad, más libre de los imperativos que imponen el retrato y las pinturas de devoción.

El asunto está tomado de las Metamorfosis de Ovidio (XIV, 623). Se relata el empeño del joven Vertumno por acercarse a la hermosa Pomona, diosa de los frutos que no cedía a los deseos de sus múltiples pretendientes, interesada sólo por el cultivo de campos y jardines. Para persuadirla de la sinceridad de su amor, Vertumno decide disfrazarse de anciana y hablarle en confidencia. Dice el texto que "se ciñó las sienes con una cofia bordada y apoyándose en un bastón y colocándose canas en las sienes fingió ser una vieja y entró en el cuidado jardín”. Nótese cómo el pintor, siguiendo al detalle la fuente literaria, prestó atención a la cofia de la vieja y se esmeró en la ejecución de las franjas deshilachadas.

Ambientó la escena en el jardín de una villa italiana, con estatuas en la perspectiva de la derecha. Pomona está sentada a los pies de un árbol desplegando su cándida belleza. Con la hoz (su más frecuente atributo que lleva en la mano derecha como apunta Ovidio ${ }^{12}$ ) proyecta sin duda cortar la mata de cardos que crece en el suelo a su lado, pues prefiere los jardines cuidados a la naturaleza salvaje. Quizá no es fortuita la elección de esta planta que en la tradición alemana se

offerirte mir jährlich 50 Sterling Gage zu geben, aber ich bedankte mich un sagte, dass ich es vor ein grosses Glück jchasste, bei ihm noch zu lernen, welches er mir in der Unterweissung gar wohl eingebracht hat." [Wackernagel, op. cit. 1895 , p. 231].

11 Vid. Nieden, op. cit. 2002, cat. nos. 4, 18, 19, 20, 22, 24, 30, 42. Véase también nota 18 más abajo.

12 "nec iaculo gravis est, sed adunca dextera falce" (su diestra no sostiene una jabalina, sino una curva hoz) [Ovidio, Met, XIV, 627]. 
entiende como símbolo de "Mannestreue"13, o confianza entre los seres humanos: una especie que la diosa no cultiva y se esfuerza en aniquilar.

Pomona vuelve el rostro al otro lado, donde está, encorvado y apoyado en un bastón, el disfrazado Vertumno, persuadiéndola: "Si eres lista, -le dice- si quieres una buena alianza y estás dispuesta a escuchar a esta vieja, que te ama más que todos ésos, más de lo que tu piensas, rehúsa todo matrimonio vulgar y elige a Vertumno por tu compañero de lecho. Aquí me tienes también como fiador de él; porque ten la seguridad de que no se conoce él a sí mismo mejor que yo lo conozco; y no anda errante por doquier a través del mundo entero; habita estos vastos parajes; y no hace como la mayor parte de tus pretendientes, amar a la última que han visto: tu serás su primera y su última pasión y sólo a ti consagrará sus años". La anciana argumenta con la mano abierta y en escorzo hacia el espectador, en un gesto de elocuencia.

No hay mención en el texto de Ovidio de la presencia de amorcillos, pero no sorprende su inclusión en estos episodios amatorios. En cambio, sí llama la atención el amorcillo oscuro y con garras en los pies que revolotea junto al hermoso y de rubios bucles. Nos preguntamos qué significado tiene tal contraposición. Quizá, haciendo eco de las palabras de Vertumno, estos amorcillos tengan una intención diferenciadora: el amor sincero de Vertumno frente al vil de los demás pretendientes. Se transmite la dual visión del amor sagrado y eterno, frente al profano, efímero y temporal. El pintor conoce bien la Emblemática, por lo que no debe sorprender una alusión de esta naturaleza ${ }^{14}$.

En cualquier caso, estos amores de naturalezas tan opuestas acechan a la diosa Pomona, que escucha pudorosa las palabras de la anciana. La palma y el pañuelo con flores de los amorcillos son símbolos alusivos a la perseverancia y a la virtud ${ }^{15}$. En el desenlace de la historia previsto por Ovidio, después de hablarle largamente y besuquearla, Vertumno terminará por quitarse el disfraz, mostrando su verdadero rostro y dejando a Pomona subyugada.

El pintor se sirvió de dos diagonales, con cruce en la cabeza de Pomona, para estructurar la composición. A la izquierda, el ramaje del árbol y, a la derecha, un paisaje con domino del elemento vertical. Las dos figuras quedan inscritas en una forma piramidal con ritmos circulares en su interior, y contrastan con el tono neutro de la vegetación, prolongada en el fondo.

Las carnaciones de Pomona retienen la atención del espectador. El pintor se esmeró particularmente en su ejecución. El rostro de ojos bajos, la boca entreabierta y los ritmos sinuosos de los brazos y del cabello, cayendo suelto sobre un hombro y en tirabuzones sobre el otro, así como las formas ondulantes de los mantos, acentúan su sensualidad. Muy delicado es el tratamiento de los detalles de su atuendo: la diadema, las perlas del cabello, la joya del brazalete y la lámina de la hoz, cuyos brillos y texturas logra con toques más empastados. Destaca la descripción de los cardos, denotando la habilidad del pintor para la naturaleza muerta: un signo de fascinación botánica que sin duda respiró en su familia, donde pudo tener al alcance fidedignos grabados.

La factura lisa en Pomona contrasta con la del rostro de la anciana, que el pintor trató diferentemente, insistiendo en las marcas de la vejez: las comisuras de los labios de boca desdentada, las carnes flácidas del cuello, las venas abultadas bajo la piel, los ojos cansados y las arrugas de la

${ }^{13}$ Con este significado lo vemos, por ejemplo, en el Autorretrato de Durero en el Louvre [Vid. TERvarent, G. DE, Attributs et symboles dans l'Art profane, 1450-1600. Dictionnaire d'un langage perdu, Ginebra, 1958, p. 298 (panicaut)].

${ }^{14}$ Sin duda por influencia de su padre, que ilustró las obras de Robert Fludd y la Emblemata de ZiNCGREF, J. W. (Fráncfort, 1619); Wüthrich, op. cit. 1966-1995, vol. II, p. 108. Alusiones de esta índole se ven también en sus retratos, a juzgar por el asunto de esta conferencia [RonNICK, M. V., "Stoic Elements in the 17th Century Portrait of Zacharias Stenglin by Matthäus Merian the Younger," Annual Papers to Graduate Student Conference, University of Pennsylvania, Philadelphia, noviembre, 1989].

15 Tervarent, op. cit., 1958, pp. 192, 296. 
frente, acentuadas con pinceladas cargadas de materia. En esto coincide con recursos técnicos de Peeter Thijs, que insiste igual en las arrugas de sus ancianos, al tiempo que emplea una factura lisa, cubriente y esmaltada en las figuras femeninas ${ }^{16}$. El modelo recuerda también prototipos de su maestro Sandrart, que se interesó de cerca en la representación de la ancianidad ${ }^{17}$.

Muy personal es la manera de articular y retorcer los paños, tendencia que observamos igual en la Artemisa de la Universidad de Missouri (fig. 3$)^{18}$, con la misma morfología del rostro del paje en segundo plano.

Merian aplicó en Vertumno y Pomona las enseñanzas de su padre y de su maestro ${ }^{19}$; pero el impacto de Van Dyck, muy patente en sus retratos, se revela también con fuerza en esta composición. La elegancia y el abandono del cuerpo son incondicionales signos vandyckianos. El rostro de la diosa deriva de los modelos femeninos de Van Dyck en el segundo periodo de Amberes,

16 Por ejemplo, San Jerónimo y el ángel, de colección privada de Barcelona [MAUfort, D., "Une Vanité avec trois Parques au Musée d'Art et d'Histoire de Genève et l'œuvre du peintre anversois Pieter Thijs (1624-1677)", Genava, Revue d'Histoire de l'Art et d'archéologie du Musée d'Art et d'Histoire de Genève, XLIX, 2001, p. 10, fig. 6]; Dédalo e Ícaro de la Bayerische Staatsgemäldesammlungen (Inv. 1975), el Sacrificio de Abraham de Iglesia de Sint Jakob de Amberes [HaIrs, M. L., Dans le sillage de Rubens. Les Peintres d'Histoire anversois au XVIIème siècle, Lieja, 1977, p. 272; MAufort, D., De Antwerpsche kunstschilder Peeter Thiijs de Oude. Een enadering aan de hand van zjn historiestuken, Tesis de Licenciatura inédita de la Universidad Católica de Lovaina bajo la dirección del profesor H. Vlieghe, 1986, p. 69, cat. n. ${ }^{\circ}$ R-9], el Lavatorio de la Iglesia parroquial de Bribir en Croacia [Tomic, R., "Prijedlog za Pietera Thysa: Pranje nogu in Bribiru", Perystil, XXXVIII, Zagreb, 1995, pp. 117-120], o el Martirio de San Pedro de la Iglesia de San Pedro en Berlaar [Vliegue, H., "Nieue toegeschrijvingen aan Antwerpse schilders uit de zeventiende eeuw", Gentse bijdragen tot de kunstgeschiedenis en de oudheidkunde, 20, 1967, p. 165; MAUFORT, op. cit. 1986, p. 84, cat. R-14].

17 Por ejemplo, la Pescadera del Herzog Anton Ulrich Museum de Brunswick, Inv. n. ${ }^{\circ} 553$, lienzo firmado y fechado en 1644, la Santa Ana en la Huida a Egipto del Museo de Bellas Artes de Rennes, el Retablo de Santa Ana de la Catedral de Salzburgo (Klemm, C., SANDrarT, Joachim von: Kunst-Werke und Lebens-Lauf, Berlín 1986, cat. 130) la Inmaculada Concepción de María de la Catedral de Freising (fechado 1678, Klemm, op. cit. 1986, cat. n. ${ }^{\circ}$ 143, p. 296) y el Retablo de la Sagrada Familia de la parroquia de San Esteban de Lambach, en el que Merian participó.

18 No veíamos motivos para dudar de la autoría de Merian en esta versión de la Universidad de Missouri [Lienzo, $95,8 \times 87,6 \mathrm{~cm}$. Museum of Art \& Archaeology, University of Missouri, Columbia, Inv. N. ${ }^{\circ}$ 66.348]; rechazada por Nieden del catálogo del pintor [NIEDEN, op. cit. 2002, p. 177, n. 42 (bajo el epígrafe "Unsichere Zuschreibungen”)], y considerada como copia del ejemplar, firmado y fechado en 1655, en la Galería de Dessau [Lienzo, 133,3 × 120,3 cm, "MATTHAEVS. MERIAN. PINXIT. A. ${ }^{\circ}$ 1655", Anhaltische Gemäldegalerie, Dessau] [Nieden, op. cit. 2002, p. 154, n. $\left.{ }^{\circ} 22\right]$. Nuestras sospechas se confirmaron al ponernos en contacto con el museo americano, que nos informó del hallazgo de una inscripción al retirar el reentelado del lienzo, en 1966: "Math [sic] Merian Junior / fecit A. ${ }^{\circ} 1647$ ". Esto aporta una evidencia material a lo observado en calidad y estilo, confirmando su autenticidad, y probando que de ésta deriva el cuadro de Dessau y no al contrario como se creía. Parece claro que Nieden no conocía la inscripción que, de hecho, se debe a la mano del propio Merian, pues coincide la grafía con la del lienzo que nos ocupa. En cuanto a la iconografía, se tiene en el museo americano como "Sofonisba" [Cf. Sовотік, K., Central Europe 1600-1800, Cat. Exp. Sarasota, Florida, The John \& Mable Ringling Museum of Art, 1972, p. 15, n. ${ }^{\circ}$ 18, il p. 53]. No obstante, a tener en cuenta la citada en la venta de la colección del hijo del pintor: "Von Matt. Merian die Arthemisia. Original" [Cf. HoET, op. cit. 1752, part II, pp. 344-357], y la alabada por Sandrart en la colección de WeRnER, J., en Ausburgo [Cf. SANDRART, in Peltzer, p. 202, citado por NIEDEN, p. 155, nota 76], lo que precisa la interpretación del asunto. Debe tratarse de los originales de Missouri y Dessau, sin que sepamos a cuál de ellas se refiere la antigua documentación. La pintura de Missouri se atribuyó primeramente a Antoine Pesne, y después a Justus Sustermans. Nuestro agradecimiento a Mary Pixley y Jeffrey B. Wilcox, del Museo de Missouri, por su amable comunicación y por habernos facilitado una buena fotografía de la obra.

19 Sin duda recordaría Merian la lección de Joachim van Sandrart, que así aconsejaba: "Represéntate en la imaginación, algunas líneas iguales, con el fin de contemplar lo que dibujas: así las lineas preparatorias te darán un conocimiento exacto de la cosa en sí. Esto debe observarse en cada empresa, incluso en la copia de estudios antiguos" [SANDRART, op. cit. 1675, p. 103]. A los pliegues acordaba una importancia esencial, y aconsejaba la utilización de maniquís y modelos de cera vestidos de telas o papel mojado, proporcionando reglas para restituirlos con belleza en función de la luz, del movimiento y de la naturaleza de las telas [Ibidem, I, 1. a parte, libro 3, cap. 12, p. 82. ${ }^{a}$. Las manos -recomendaba- no deben sobrepasar la altura de la cabeza ["Eines zierlichen Bildes Hand /soll nicht höher als der Kopf (...) erhoben seyn”; Ibidem, p. 103]. 


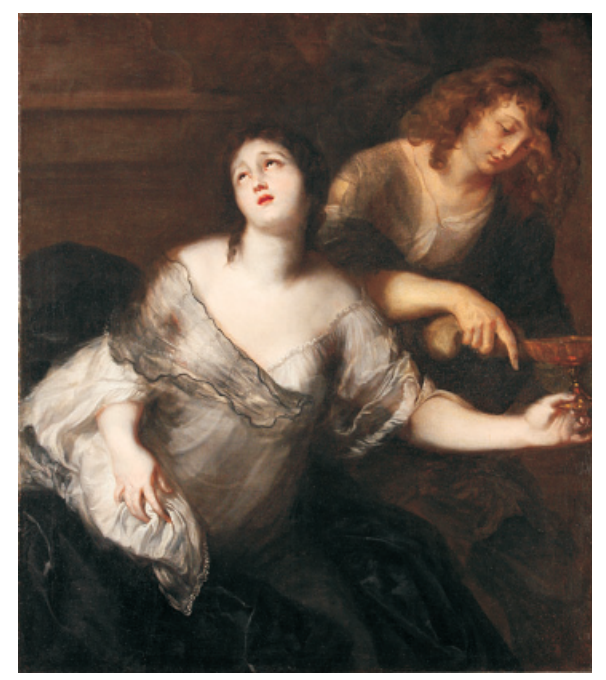

Fig. 3. Matthäus Merian el Joven. Artemisa. Lienzo, 95,8 × 87,6 cm. Museum of Art \& Archaeology, University of Missouri, Columbia.

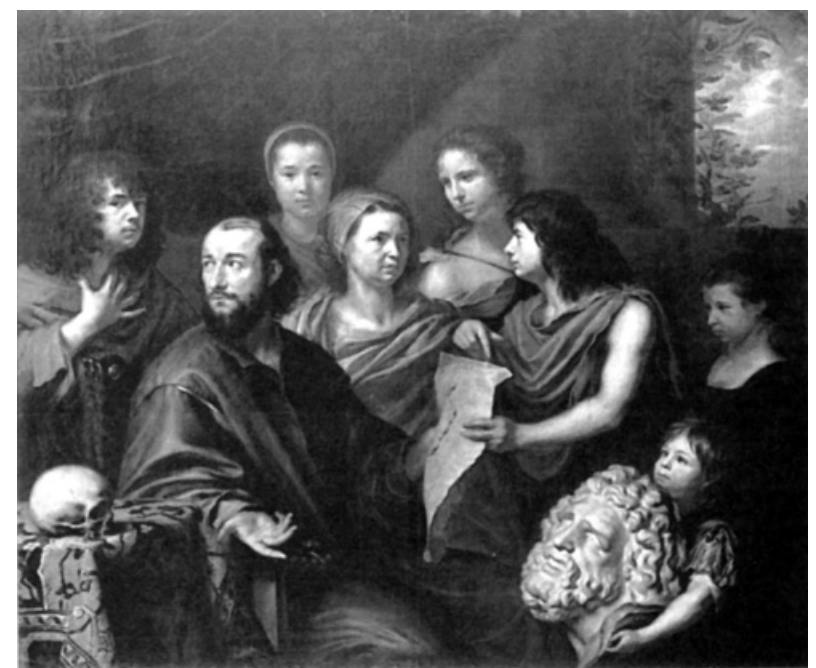

Fig. 4. Matthäus Merian el Joven. Retrato de la Familia Merian. Lienzo, 118,5 × 140. Basel Kunstmuseum.

semejante a la Virgen en los Desposorios del beato Hermann Joseph, y a Venus desarmando a Marte, composición con la que comparte el mismo espíritu ${ }^{20}$. El torso desnudo no es ajeno en el retrato de Katherine Manners junto al duque de Buckingham como Venus y Adonis, lienzo de Van Dyck que había quedado en el taller londinense ${ }^{21}$ y que sin duda Merian conocería.

Por último, la palma de la vieja, avanzando abierta hacia el espectador y colocada como aquí en un lugar central, es recurso con raíces venecianas utilizado por Van Dyck en varias pinturas desde su juventud ${ }^{22}$, como en la Lamentación del Museo de Amberes ${ }^{23}$, en San Juan Bautista de los Dos Santos Juanes ${ }^{24}$ y en retratos como el del Marqués de Leganés ${ }^{25}$. Es probable que

20 También similar en la Visión de San Antonio de Padua de la Pinacoteca de Brera, o la Virgen con Niño o los Desposorios de Santa Catalina de la colección real inglesa, o a la Virgen con Niño y Santa Catalina del Metropolitan de Nueva York [Véase Barnes, S. J., Poorter, N. de, Millar, O. \& Vey, H., Van Dyck: A complete Catalogue of the Paintings, Londres-New Haven, 2004, nos. III.10, III.38, III.43, III.44, III.49, III.A12].

21 Se ha señalado también la posibilidad de que se trate del lienzo con Phillis y Corridon que se registra en su colección [Brown, C. \& RAMSAY, N., "Van Dyck's collection: some new documents", Burlington Magazine, vol. 132, 1990, pp. 707, 709; BARNES et al., op. cit. 2004, p. 137, n. ${ }^{\circ}$ I.158].

22 Por ejemplo, en la Serpiente de metal del Museo del Prado; en el Milagro de los panes y los peces de Sanssouci (perdido en la Segunda Guerra Mundial); en San Martín entregando su capa al pobre de la colección real inglesa; en diferentes versiones del Martirio de San Sebastián (Múnich y Mallorca), y la Continencia de Escipión de la Christ Church de Oxford; [BARNES, et al., op. cit. 2004, nos. I. 3; I. 12; I. 39].

${ }^{23}$ BARNes, et al., op. cit. 2004, p. 269, n. ${ }^{\circ}$ III.30.

24 El lienzo de gran tamaño se perdió en Berlín en la Segunda Guerra Mundial [BARNES, et al., op. cit. 2004, p. 52 , n. ${ }^{\circ}$ I.37]. El boceto o modelletto está en la Real Academia de San Fernando de Madrid [Díaz Padrón, M., Pedro Pablo Rubens (1577-1640), Exposición Homenaje, Madrid, 1977-1978, p. 53, n. ${ }^{\circ}$ 27], y dos dibujos previos en la Fundación Custodia de París [Jaffé, M., "The Second Sketch Book by Van Dyck", Burlington Magazine, 1959, p. 321, figs. 14 y 15; Vey, H., Die Zeichnungen Anton van Dycks, Monographen des "National Centrum voor de Plastische Kunsten Van XVI de en XVII de Eeuw, Bruselas, 1962, I, p. 120, n. ${ }^{\circ}$ 50].

${ }^{25}$ Díaz Padrón, M., "Infortunio y fortuna de los retratos de Don Diego de Mesía, Marqués de Leganés", Anales de Historia del Arte, volumen extraordinario 2008, pp. 189-212. 
viera dibujos de estas composiciones en el taller de Van Dyck. Debió gustar a Merian este gesto, pues lo repitió idéntico al pintar a su padre en el Retrato de familia de la Kunsthaus de Basilea (fig. 4) ${ }^{26}$, donde incluyó también su propio rostro, a la izquierda, mirando al espectador.

Es afortunada la vista de la derecha, con la perspectiva de un jardín flanqueado por álamos, un palacio y una estatua vista de perfil, que capta con pincelada suelta y segura. Merian reproduce esculturas análogas en el Martirio de San Lorenzo (1648) y en la Decapitación de Santa Catalina (1652) de la Catedral de Bamberg ${ }^{27}$, donde persisten recursos vandickyanos ${ }^{28}$. El gusto por la estatuaria debió cultivarlo junto a Sandrart quien, como es conocido, produjo numerosos grabados de estatuas antiguas. Algunos dibujos conservados en Berlín y párrafos de la Autobiografía de Merian, reflejan su interés por la estatuaria, la arquitectura y los jardines ${ }^{29}$. No obstante, la atmósfera es muy afín a las obras de Peter Thijs, con las que comparte modelos, espíritu, recursos compositivos y técnicos ${ }^{30}$. El modelo de la diosa recuerda también las bellas mujeres de Thomas Willeboirts Boscchaert ${ }^{31}$, y el característico cupidillo rubio con tirabuzones, utilizado tanto por éste como por Thijs ${ }^{32}$.

${ }^{26}$ Lienzo, 118,5 × 140. Basel Kunstmuseum, Inv. N. ${ }^{\circ}$ 2318. [HÜGSEN, H., Nachrichten von Franckfurter Künstlern, Fráncfort, 1780, p. 57; NiEDEN, op. cit. 2002, p. 125-127, n. $\left.{ }^{\circ} 1\right]$. La pintura data de su vuelta a Fráncfort en 1642 . Quedó en la casa familiar, según el inventario hecho a la muerte de Matthäus Merian el Viejo, en 1650 [Cf. LÜBBECKE, F., Merians Frankfurt, Fráncfort, a.M., 1940, f. 9; Cit. NiEDEN, op. cit. 2002, p. 125].

27 Hoy en el Museo Diocesano [Nieden op. cit. 2002, p. 130, n. ${ }^{\circ} 4{\left.\text {, ill, p. 151, n. }{ }^{\circ} 20\right] .}^{2}$

${ }^{28}$ Las figuras de los esbirros son préstamo al Martirio de San Pedro de Van Dyck de Bruselas, [Konijnlijk Museum voor Schone Kunsten, Inv. n. ${ }^{\circ}$ 215; BARNES et al., op. cit. 2004, p. 59, n. ${ }^{\circ}$ I.43] y colección privada de Barcelona [DíAz PAdRón et al., Van Dyck y España, cat. n. ${ }^{\circ} 26$ (en prensa)]. Del Prendimiento de Cristo de Van Dyck, hoy en el Museo del Prado y entonces en la casa de Rubens, tomó el efecto nocturno de la antorcha que ilumina la escena, hábil recurso de Van Dyck que, curiosamente, fue lo que más valoró A. R. Mengs [Ponz, A., Viage de España, en que se da noticia De las cosas más apreciables, y dignas de saberse, que hay en ella, Tomo Sexto, Trata de Madrid, y Sitios Reales inmediatos, Tercera impresión, Madrid, 1793 (1789), VI, p. 202; DíAz PADrón, M. et al., Van Dyck y España, cat. n. ${ }^{\circ} 11$ (en prensa)].

29 "Es waren meiner Zeit viele Deutsche und Niederländer da, die in 5 oder 6 Jahren so viel gezeichnet hatten, als ich allein, als Statuen, Basserelieven und Accademie, habe mich auch in der architettura civile exerciert" [WACKERNAGEL, op. cit. 1895, p. 236]. Véanse lose Estudios de estatuas y la vista del Templo de Vesta en Tivoli del Kupferstichkabinett de Berlín [NIEDEN, op. cit. 2002, pp. 200-2001, cat. Nos. 76-78].

30 Véase el Baño de Betsabé de Peeter Thijs [Lienzo, 197,5 × 242,5 cm. Firmado en la balaustrada: "P. THYS". Gateshead, Shipley Art Gallery, cat. B9984 [Maufort, op. cit. 1986, p. 156, cat. R. 39; Douglas Stewart, J., "Peter Thijs (1624-1677). Recovering a scarcely known Antwerp painter", Apollo, vol. CXLV, 1997, p. 41]. A pesar de la firma, esta pintura y un dibujo preparatorio se mantienen con atribución a Thomas Willeboirts Bosschaert en la monografía de A. Heinrich [Thomas Willeboirts Bosschaert (1613/14-1654). Ein Flämische nachfolger Van Dycks, Turnhout, 2003, p. 237, cat. A.50, ill. 77]. Véase también la similar tipología de los modelos femeninos y concepción de los pliegues de los mantos de las Parcas y el Tiempo [Lienzo, $274 \times 195 \mathrm{~cm}$. Grenoble, Musée des Beaux Arts (Inv. MG106). Maufort, op. cit. 1986, p. 192, n. ${ }^{\circ}$ M21; MAUfort, op. cit. 2001, pp. 3-15 (como P. Thijs). La atribución de esta pintura a Th. Willeboirts Bosschaert persiste sin motivos en la monografía citada [Heinrich, op. cit. 2003, kat. N. ${ }^{\circ}$ A30a]. La posición de la anciana está en la Santa Ana de Peeter Thijs en San Roco curando a los apestados de Dendermonde [Lienzo, 234 $\times 193 \mathrm{~cm}$. Dendermonde, Onze-Lieve-Vrouwekerk; MAUfort, op. cit. 1986, p. 118, cat. n. ${ }^{\circ}$ R26].

31 El rostro de ojos bajos se asemeja a la Virgen de la Visión de San Francisco Javier de Willeboirts Bosschaert en la Galería de Múnich [Lienzo, $128 \times 150,2 \mathrm{~cm}$. Múnich, Bayerische Staatsgemäldesammlungen, Alte Pinakothek (depósito), Inv. N. ${ }^{\circ}$ 1256] [HaIRS, op. cit. 1977, p. 241; HeINRICH, op. cit. 2003, p. 199, n. ${ }^{\circ}$ A34]. De hecho, obras de Matthäus Merian el Joven se confunden con Th. Willeboirts Bosschaert. El retrato de Friedrich von Hessen-Eschwege como cazador del Castillo de Grunewald de Berlín (inv. GK I 5546) que se atribuyó al flamenco, se ha sugerido sea obra de M. Merian el Joven [HeINRICH, op. cit. 2003, p. 362, n. ${ }^{\circ}$ E66].

32 Douglas StewarT, op. cit. 1997, pp. 40, 42; Idem, "Thomas Willeboirts Bosschaert and Peeter Thijs: A Tale of Two Tangled Antwerp Painters; with an Excursus on Van Dycks's St. Felix of Cantalice”, in Van Dyck 1599-1999, Conjectures \& Refutations, Brepols, 2001, pp. 271-288. 
Curiosamente, Merian se aleja de la composición de Van Dyck del mismo asunto (hoy en el Palazzo Rosso de Génova), a pesar de que el dibujo preparatorio era de su propiedad ${ }^{33}$. Nos preguntamos si se tornaría al lienzo con Vertumno y Pomona de Peter Thijs (tabla, 91,5 × 118,8 $\mathrm{cm}$ ), hoy sin localizar ${ }^{34}$. Está en lo posible, vistas las analogías en la producción de estos pintores ${ }^{35}$, un nexo que también se deja ver en la colección reunida por Matthäus Merian, donde figura un lienzo de Peter Thijs (Venus y Adonis, hoy también sin localizar) ${ }^{36}$. Interesa de otra parte mencionar que constan en dicha venta varios retratos de Van Dyck, obras de otros flamencos y del propio Merian ${ }^{37}$.

Esto nos lleva a reconocer un contacto, que situamos en estos últimos años de la actividad de Van Dyck en Londres. Hoy se da poco crédito al supuesto viaje de Thomas Willeboirts Bosschaert por Inglaterra, Alemania y España ${ }^{38}$. No obstante, su influencia pudo llegar a Merian a través de Thijs, que trabajó con Willeboirts y probablemente fue su alumno ${ }^{39}$. En cuanto a Thijs, algunas fuentes hoy olvidadas apuntan su presencia en Londres en $1639^{40} \mathrm{y}$, pese al vacío documental al respecto del otro lado de La Mancha, la huella de Van Dyck en su obra fue tan indeleble que el hecho se da por asumido. No obstante, es mucho lo desconocido respecto a los asistentes de Van Dyck en Inglaterra y a su cometido en el taller ${ }^{41}$.

33 El dibujo fue uno de seis que llegaron al Museo de Berlín de la colección de Matthäus Merian el Joven [Konow, op. cit. 1940, p. 62 (pone en duda la atribución a Van Dyck)]. Como Rubens fue incluido en el corpus de dibujos del maestro [Burchard, L. \& D'Hulst, R. A., Rubens Drawings, Amberes, Nationaal centrum voor de plastische kunsten van de XVIde en XVIIde eeuw, 1963, n. $\left.{ }^{\circ} 76\right]$. Vey lo atribuyó a Van Dyck, relacionándolo con el lienzo de Génova [VEY, op. cit. 1962, p. 126, n. $\left.{ }^{\circ} 76\right]$, atribución que apoyó JAFFÉ, M. ["Rubens as a draughtsman", Burlington Magazine, 1965, p. 379] y que no se pone en duda en la actualidad.

${ }^{34}$ Estuvo en la colección de Jean van Lancker, muerto en 1719 en Amberes (n. ${ }^{\circ} 113$ ) [Vid. MAUFORT, op. cit. 1986, p. 227, cat. n. ${ }^{\circ}$ LM-13] y se vendió en la Chambre des Arquebusiers de Amberes, el 23 de mayo de 1769 [Duplessis, Catalogue d'un cabinet de tableaux des plus célèbres et meilleurs maîtres de l'Europe, très-bien conditionnés et dlaissés par feu Monsieur Jean van Lancker... Amberes, 1769 (620)].

35 Basta cotejar el joven que acompaña a la Artemisa de Merian en la Universidad de Missouri con los ángeles mancebos de Peeter Thijs en la Expulsión de Adán y Eva en la Galería de Stuttgart Lienzo, $183 \times 261 \mathrm{~cm}$. Stuttgart, Staatsgalerie, Inv. N. 735 [HAIRS, op. cit. 1977, p. 78 (como Jan Boeckhorts); MAUFORT, op. cit. 1986, p. 75, cat. N. ${ }^{\circ}$ R11 (como Peter Thijs)]. Y en San Francisco arrodillado ante la Virgen de la Porciúncula del Museo de Amberes [Lienzo, $248 \times 263 \mathrm{~cm}$. Amberes, Koninklijk Museum voor Schone Kunsten, inv. n. ${ }^{\circ}$ 352. [Véase HaIRS, op. cit. 1977, p. 271; StEWART, Douglas, op. cit. 2001, p. 277, fig. 8; MAUFORT, op. cit. 1986, cat. n. ${ }^{\circ}$ r16].

36 “on Teisen Venus und Adonius", colección puesta en venta en Fráncfort, en 1717 [HoEt, G., Catalogus of naamlyst van schilderyen..., La Haya, 1752, 1770, p. 344 bis, 357].

37 Catalogus derjenigen Mahlereyen so in Hern van Merian seel. Cabinet gefunden worden nunmehro bey Herrn Jacob Heldewir in Franckfurt im Verkauff zu haben sind [Hoet, op. cit. 1752/70, II, p. 344-357; GwINNER, F., Kunst und Künstler in Frankfurt am Main, Fráncfort, I 1862, p. 164f.; GERSON, H., Ausbreitung und Nachwirkung der holländischen Malerei des 17. Jahrhunderts, Haarlem, 1942, p. 265].

38 Transmitido por Houbraken según noticia de BIE, C. de [BIE, C. de, Het Gulden Cabinet van de edel vry Schilder-Const, 1661, p. 166; Houbraken, A., De Groote Schouburgh der Nederlandsche konstschilders en schilderessen, Ámsterdam, 1718/1721, I, p. 357; NiEDEN, op. cit. 2003, I, p. 20; II, p. 369, n. ${ }^{\circ}$ 76-78, p. 381, Doc 26].

39 Vlieghe, H., "Thoughts on Van Dyck's Early Fame and Influence in Flanders", in Van Dyck 350, Studies in the History of Art, vol. 46 (ed. BARnes, Susan J. \& WheElock, Arthur K., Jr.), Washington, 1994, p. 214.

40 Descamps transmite que Peter Thijs estaba en Londres en 1639 [DEscamps, J. B., La vie des peintres flamands (...), 1763, p. 561]; en el manuscrito anónimo y conservado en el Louvre sobre la vida y obra de Van Dyck se dice que viajó a Londres junto a otros colegas en 1639 para trabajar en el taller de Van Dyck y que su tarea consistía en pintar los fondos de los cuadros [LARSEN, E., op. cit. 1975, p. 80, 121]; noticia que recogen también Cust, L. [op. cit. 1900, p. 151] y MAUFORT, D. [op. cit. 1986, p. 10].

${ }^{41}$ Se sabe que Van Dyck viajó a Inglaterra acompañado de Jean de Reyn (1610-1678?), al que había formado en Amberes y en compañía de quien volvió al Continente en 1634-35. Descamps informa de que De Reyn permaneció en Londres hasta la muerte de Van Dyck [DescamPs, op. cit. 1763, pp. 27-28], noticia que recoge también Michiels, apun- 
En el caso de Matthäus Merian el Joven, podemos recrear las circunstancias que condicionaron su encuentro con Van Dyck. Como apuntamos ya, fue determinante la mediación de Michel Le Blon. Es necesario detenernos en la relevancia de este grabador, muy respetado conocedor de arte y agente suizo en la Corte británica, y en su proximidad con el joven pintor.

Matthäus Merian el Joven lo conocía bien, pues Le Blon había sido alumno de su padre y era primo de su maestro Sandrart, al que acompañó de viaje por Italia en 1627. Estaba muy bien integrado en la corte británica, como "Agente suizo" desde 1618, y "Agent de la Roÿne et Couronne de Suede chez Sa Ma ${ }^{\text {té }}$ de la Grande Bretagne" desde $1633^{42}$. Según una declaración de Edward Cooper, fue Le Blon quien recomendó Van Dyck a Carlos $\mathrm{I}^{43}$, lo que no deja de tener significación. Le Blon intervino también en la venta de pinturas de la colección de Rubens al duque de Buckingham en 1625-162744, invirtió altas sumas en compras de la colección de Rubens ${ }^{45}$ y medió en la adquisición de objetos para la reina Cristina de Suecia ${ }^{46}$. Además, se encargó de la venta en Ámsterdam de cuadros de la colección de Van Dyck, que propuso al marchante Matthijs Musson en Amberes, según testimonia la correspondencia que ha llegado a nosotros ${ }^{47}$.

Van Dyck hizo de él un retrato que hoy conserva la Galería de Ontario (fig. 5) ${ }^{48}$. Le Blon, por su parte, grabó de Van Dyck el retrato del Príncipe Charles Louis del Palatinado, hasta fechas

tando la buena ejecución de sus obras [Michiels, op. cit. 1882, pp. 358, 446, 542-544]. No obstante, algunos lienzos que hizo para Dunkerke indicarían su abandono antes de esta fecha [el Martirio de los cuatro coronados, firmado y fechado en 1640, encargo para la capilla de San Eloy de Dunkerke, así como el Retrato de Pierre de Corneille, firmado, en el Museo Lambinet de Versalles; Vid. Le Siècle de Rubens dans les collections publiques françaises, Cat. Exp. París, Grand Palais-1977-78, pp. 145-148]. Véase también Trésors des Musées du Nord de la France. III. La peinture flamande au temps de Rubens, Cat. Exp. Lille-Calais-Arras, Musée des Beaux Arts, 1977, pp. 103-106; HAIRs, op. cit. 1977, p. 33]. En su trabajo sobre Van Dyck y sus discípulos, Michiels prestó también atención al inglés William Dobson (16101646) llamado por Carlos I "el Tintoretto de Inglaterra" y sucesor de Van Dyck como pintor oficial de corte. Remigius van Leemput (1607-1675) entró en el taller de Van Dyck en 1628 y fue admitido como maestro de la guilda el año siguiente, partiendo para Inglaterra después del maestro en 1632; a Jacques Gandy (1619-1689), que partió a trabajar a Irlanda y cuyos retratos de su mano del castillo de Kilkenny se vendieron como originales de Van Dyck; al miniaturista Jean Petitot, a quien Van Dyck dio lecciones de pintura por encargo de Carlos I; a David Beck (1625-1656), que Van Dyck inició en el colorido. Menciona entre los que frecuentaban el Club de San Lucas: Adriaen Henneman, Jean van Belcamp, Theodore Russel, Edouard Pierce, Gérard Pietersz van Zyl, Bertrand Fouchier, Anne Carlisle y Henri Stone [MichIELS, op. cit., 1882, pp. 545-563].

42 Título que recuerda el grabado en la Iconografia de Van Dyck [The New Hollstein. Ducth \& Flemish Etchings, Engravings and Woodcuts 1450-1700. Anthony Van Dyck (ed. Turner, S. \& DePAuw, C.), Rotterdam, 2002, VI, n. $\left.{ }^{\circ} 467\right]$.

${ }^{43}$ Vertue, G., "Notebooks", Walpole Society, 1930-55, vol. I, p. 29; vol. II, p. 98.

44 Müller, J. M., Rubens. The artist as a Collector, Princeton, New Jersey, 1989, p. 78.

45 Denucé, J., Na Peter Pauwel Rubens. Documenten uit den Kunsthandel te Antwerpen in de XVIIe eeuw van Matthijs Musson, Amberes, 1949, p. XVI.

${ }^{46}$ Un salero de marfil con el Nacimiento de Venus diseñado por Rubens y ejecutado por Georg Petel, hoy en el Palacio de Estocolmo [Véase MülLER, op. cit.1989, p. 146, pl. 119].

47 Véase las cartas de Le Blon a Matthijs Musson, 22-IX-1644, 15-VI-1645, 24-VII-1645 [DENUCÉ, op. cit. 1949, pp. 20, 33, 34].

${ }^{48}$ Lienzo, 78,1 $\times 61 \mathrm{~cm}$. Art Gallery of Ontario, Toronto, inv. 64/65. En cuanto a la datación de este retrato, se ha propuesto por razones estilísticas el segundo periodo de Amberes de Van Dyck [BARNES, et al., op. cit. 2004, p. 321, n. ${ }^{\circ}$ III.93], y la fecha de 1630-35 [Handbooks, Catalogue illustré, Toronto, 1974, p. 50; Art Gallery of Ontario Selected Works, Toronto, 1990, p. 87]. Se cree que la pintura estuvo en 1734 en la venta de Willem Six, Amsterdam, 12-V-1734, n. ${ }^{\circ} 30$ [De la Fontaine-Verwey, 1969, p. 119; Larsen, E., The Paintings of Anthony van Dyck, Freren, 1988, n. ${ }^{\circ}$ 542;]. Theodor Matham lo reprodujo en grabado con inscripción relativa al título de sus funciones [véase nota 44]. Existe copia en Ámsterdam [lienzo, $57 \times 46 \mathrm{~cm}$. Vid. All the paintings of the Rijksmuseum in Amsterdam, Maarsen, 1976, p. 210, n. ${ }^{\circ}$ A205; Larsen, op. cit. 1988, n. $^{\circ}$ A104]. 


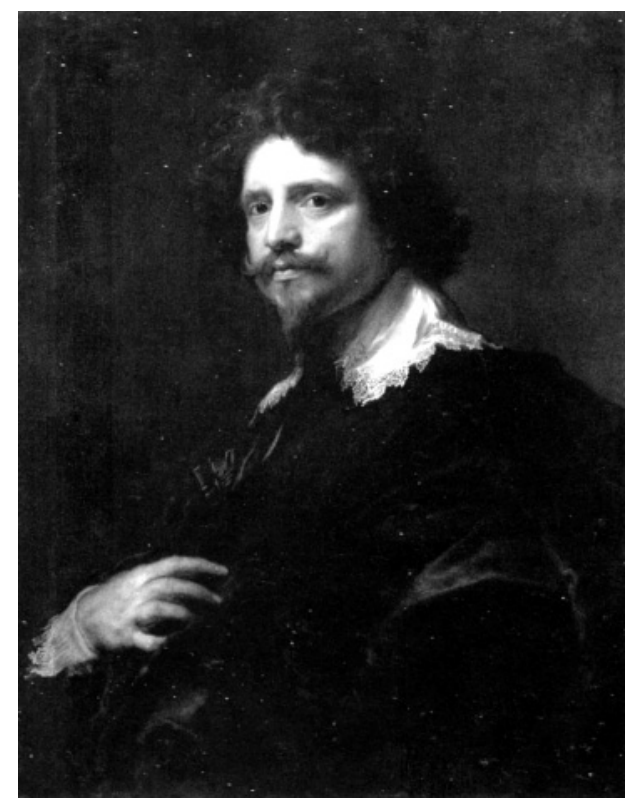

Fig. 5. Anton van Dyck. Retrato de Michel Le Blon. Lienzo, 78,1 × $61 \mathrm{~cm}$. Art Gallery of Ontario, Toronto.

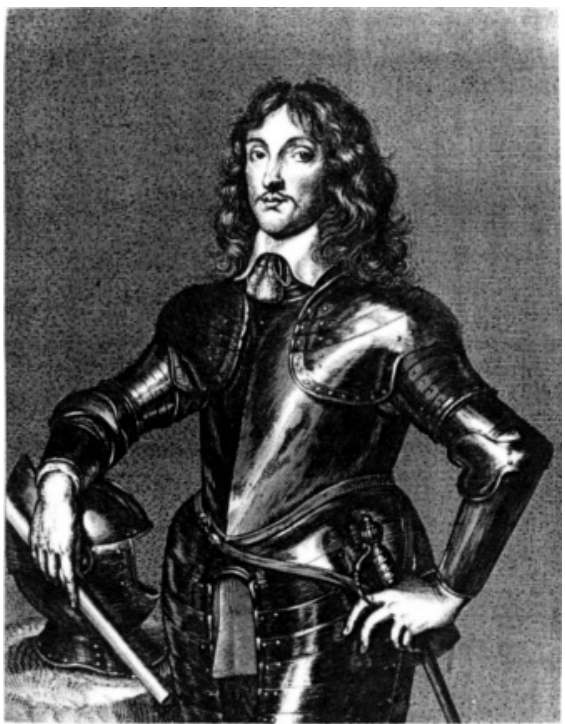

SFRINIS FTCEIST PRINCEPS AC DOMINVS DN CAROLVS

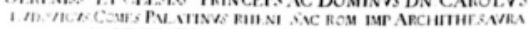

Fig. 7. Michel Le Blon según Van Dyck. Retrato del Príncipe Charles Louis del Palatinado. Grabado.

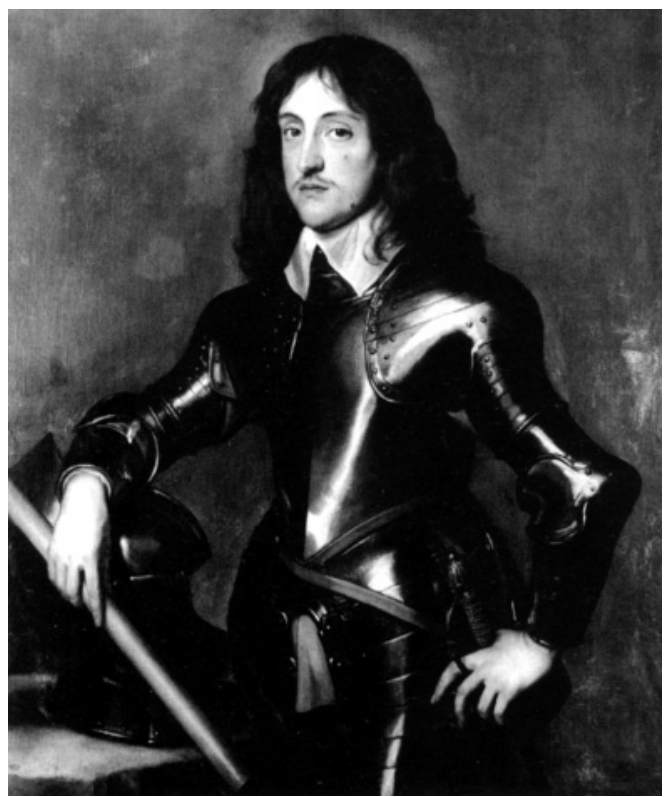

Fig. 6. Antón van Dyck y colaborador. Retrato del Príncipe Charles Louis del Palatinado. Paradero actual desconocido.

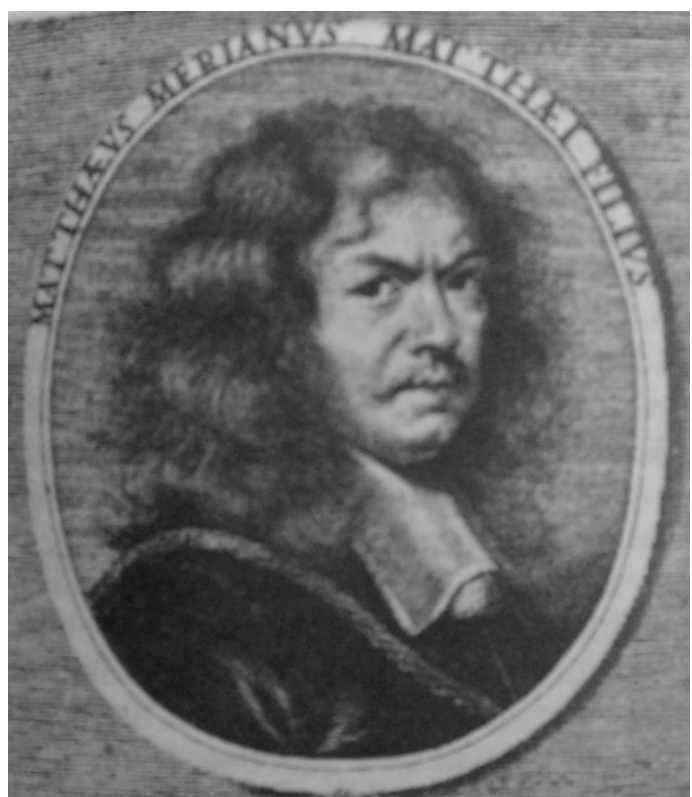

Fig. 8. J. von Sandrart. Retrato de Matthäus Merian el Joven. Teutsche Academie (1675). 
recientes en el Palacio de Marienburg (fig. 6) ${ }^{49}$. De este retrato - que se dice fue pintado ad vivum en Londres en $1641^{50}$ - se subraya la excelente calidad de la cabeza y las manos; y se apunta la posibilidad de que los accesorios fueran pintados por algún asistente ${ }^{51}$. Ciertamente, la falta de proporción entre la cabeza y el busto armado conduce a ver dos manos en su ejecución. Cabe preguntarse si sería Merian, aún en Londres junto a Van Dyck en esta fecha, el responsable de terminarlo. No sería extraño a la vista de retratos de Merian como el de Karl Gustav Wrangler en el Palacio de Stoklosters o el del Emperador Leopoldo I a caballo del Kunsthistorisches de Viena. Quizá esto explique que Le Blon grabara un Van Dyck en el que habría participado su protegido (fig. 7) ${ }^{52}$.

Auspiciado pues por Michel Le Blon entró Merian a los dieciocho años en el taller de Van Dyck. Por su familia materna tenía el joven ascendencia flamenca. Su abuelo, Theodoor de Bry, había vivido en Amberes y en Londres antes de instalarse definitivamente en Fráncfort ${ }^{53}$. Su padre, admirador y grabador de obras de Peter Paul Rubens ${ }^{54}$, lo alentaría a perfeccionar su formación junto al mejor discípulo del genio flamenco de su generación. Sin duda pesaría también en favor de Merian el haber sido alumno privilegiado de Sandrart ${ }^{55}$. Éste lo había tomado como alumno en Fráncfort (hacia 1636-1637), llevándolo consigo y su familia al partir a Holanda. Sabemos que se detuvieron a visitar a Abraham Bloemaert en Utrecht ${ }^{56}$ y suponemos que, llegados a Ámsterdam, el discípulo respiraría el ambiente artístico y coleccionista en el que Sandrart estaba bien introducido. Merian lo ayudó en los encargos de retratos y en los grandes retablos para la Abadía de Lambach ${ }^{57}$.

El teórico alemán, cuya relación con Rubens es conocida ${ }^{58}$, cuenta que el joven Merian conoció a Rubens y a Jordaens, y confirma la relación de confianza y familiaridad que tuvo con Van Dyck $^{59}$. De esto dejo constancia en la Teutsche Academie (1675), junto al retrato de su discípulo

49 Lienzo, $107,3 \times 92,7 \mathrm{~cm}$. Nordstemmen, Schloss Marienburg [BARNES et al., op. cit. 2004, p. 487, n. ${ }^{\circ}$ IV.72]. Pasó recientemente en subasta (Sotheby's, Londres, 7-XII-2005, n. ${ }^{\circ} 26$ ). El retrato fue grabado primeramente por Le Blon (1652) y después por Samuel Bernard (1657) [New Hollstein, op. cit. 2002, V, 422 y 423].

${ }^{50}$ El príncipe llegó a Londres en el mes de marzo, permaneciendo en la corte británica hasta el verano, con corta estadía en Escocia [Loomie, A. J. (ed.), Ceremonies of Charles I, Nueva York, 1987, pp. 304, 313].

${ }^{51} \mathrm{Al}$ no llevar el retratado el collar de la Orden de los Jarreteros se piensa que Van Dyck lo dejó inacabado [Cit. BARNES et al., op. cit. 2004, p. 488].

52 Hollstein, New, op. cit. 2002, V, 422.

${ }^{53} \mathrm{Su}$ madre era nieta de Johan Theodor de Bry, grabador y editor nacido en Lieja y con residencia en Amberes y en Londres antes de instalarse definitivamente en Fráncfort (1588).

${ }^{54}$ Grabó, entre otras, escenas del ciclo de la Vida de María de Medicis y el retrato del Conde-Duque de Olivares [BAUDouIN, F., "Peter Paul Rubens und Matthaeus Merian der Ältere", Zeiftschrift fur Kunstgeschichte, 1990, pp. 160-176].

55 Sandrart le enseñó gratuitamente durante dos años, por lo que se supone que tenía el privilegio de "Meistersohns" ["Allda whonte ich bei meiner Herrschaft, bis er mir a. ${ }^{0} 1639$ in September meine noch übrige Lehrzeit schenkte" [WACKERNAGEL, op. cit. 1895, p. 231; TACKE, A., "Das tote Jahrhundert. Anmerkungen zur Forschung ubre die deutsche Malerei des 17. Jahrhunderts", Zeitschrift des Vereins für deutsche Kunstwissenschaft, 51, 1997, p. 50; NIEDEN, op. cit. 2002, p. 25].

${ }^{56}$ SANDRART, op. cit. 1675 , I, 2. ${ }^{\text {a }}$ parte, cap. 17, p. 298.

57 Heck, M. C., Théorie et pratique de la peinture. Sandrart et la Teutsche Academie, 2006, p. 33; Idem, "D'une école de peinture à une académie de papier: les retables de l'église de Lambach”, in Joachim von Sandrart zum 400. Geburtstag: ein europäischer Künstler und Theoretiker zwischen Italien und Deutschland, Actas del Congreso Internacional de Roma, Bibliotheca Hertziana, abril 2006 (publicación anunciada en 2008).

58 SANDRART, op. cit. 1675, I, 2. a parte, libro 3, cap. 16, p. 291; HeCK, M. C., "Le regard paradoxal de Joachim von Sandrart sur Rubens", in Le Rubénisme en Europe aux XVIIe et XVIIIe siècles, Akten des internationalen Kolloquiums in Lille-Arras-Antwerpen, abril 2005, Turnhout 2005, pp. 183-196;

59 “... sich auch nach Engeland verfügt, alles ruhmwürdige am Königlichen Hof besichtiget und mit dem Antonio van Dick Verträulichkeit und Familiaität aufgerichtet, sich auch zu Antorf mit Rubens, Jordans und de bästen Künstlern bekant gemacht" [SANDRART, op. cit. 1675, in Peltzer, op. cit. 1925, p. 200]. 
ya maduro (fig. 8). Él mismo había estado en Londres en 1628, para ayudar a Honthorst en los encargos de Carlos I. Allí conoció a Íñigo Jones y recorrió las colecciones del rey en Whiteha11, las del conde de Arundel y las del duque de Buckingham ${ }^{60}$. Sin duda, Sandrart fue también parte activa en promover que el discípulo trabajara con Van Dyck, pintor que admiraba ${ }^{61}$ y del que poseyó un retrato: el del escultor Georg Petel (hoy en la Alte Pinacothek de Múnich) ${ }^{62}$, que introdujo en grabado de la Teutsche Academie ${ }^{63}$.

En fin, éste es el entramado en que la intervención de Le Blon, a quien Sandrart se refiere en ocasiones como "nuestro mecenas" ${ }^{4}$, será decisiva en este cruce de destinos. Llegado a Londres, Merian debió visitar también los gabinetes de los coleccionistas, y estar en contacto con Lord Arundel, pues conocía bien su colección ${ }^{65}$. Sería en el taller de Van Dyck donde trataría con Peeter Thijs, lo que explicaría la relación en sus obras.

M. Merian no proporciona muchos detalles sobre su estancia en Londres, pero sí especifica que no habitaba la casa de Van Dyck ${ }^{66}$. ¿Se referiría entonces a M. Merian y a P. Thijs el documento (del 20 de enero de 1641) que habla de unos sirvientes de Van Dyck, de la Casa de Lady Carlisle, que "estaban allí para ver pinturas"67?

En cualquier caso, su ayuda sería inestimable, considerando el escaso tiempo que tendría el maestro para pintar en estos dos últimos años de su vida en que, al margen de los numerosos encargos que le acuciaban en Londres, realizó varios viajes al Continente, contrajo matrimonio con Mary Ruthveen, y la enfermedad le impedía concluir los retratos de los Oranje ${ }^{68}$.

Matthäus Merian el Joven y Peeter Thijs debieron suponer una ayuda valiosa para concluir los retratos de Van Dyck. La calidad que advertimos en sus pinceles avala tal capacitación y explica la confusión de sus obras. Creemos de utilidad tener en cuenta a Matthäus Merian el Joven en el círculo de Van Dyck, y reconocer su obra en su expresión más personal, para desligarla de la del

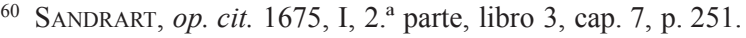

${ }^{61}$ Del arte de Van Dyck valora especialmente la delicadeza [SANDRART, op. cit. 1675, 1. a parte, Libro 3, prefacio dedicado a los jóvenes pintores, p. 58].

${ }^{62}$ BARNES et al., op. cit. 2004, n. ${ }^{\circ}$ III.117.

${ }^{63}$ Sandrart, op. cit. 1675, p. 355; New Hollstein, IV, p. 354; véase el dibujo en Feuchtmayr, K. et al., 1973, Berlín, n. ${ }^{\circ} 162$.

${ }^{64}$ SANDRART, op. cit. 1675, in Peltzer, op. cit. 1925, p. 244.

65 Así lo revela su Autobiografía [WACKernagel, op. cit. 1895, p. 231. D. Nieden sugiere que pudo conocerla a través de Wenzel Hollar, próximo a Arundel [NIEDEN, op. cit. 2002, p. 25].

66 "weil ich bei ihm nicht logierte, auch nur des Mittags bei seinem hossmeister" [WACKERNAGEL, op. cit. 1895, p. 231].

${ }^{67}$ Wood, "Van Dyck and the Earl of Northumberland: Taste and Collecting in Stuard England", Van Dyck 350, op. cit. 1994, p. 310.

${ }^{68}$ De principios de 1639 datan varias órdenes de pago al pintor por pinturas "para Su Majestad" [CARPENTER, W. H., Pictorial Notices, Consisting of a Memoir of Sir Anthony Van Dyck..., Londres, 1844, p. 74], y a finales de año otros documentos muestran que promete el retrato de Sir Edmund Verney [Verney, P., Memoirs of the Verney Family during the Civil War, Londres, 1892, I, pp. 257-261], y pagos por pinturas para el duque de Northumberland [WooD, J., op. cit. 1994, p. 309]. Al año siguiente contrae matrimonio con Mary Ruthveen y para el 8 de octubre, ya muerto Rubens, está en Bruselas para el día de San Lucas [Según carta del cardenal infante a Felipe IV, Rooses, M. \& RuELENS, Ch., Correspondance de Rubens et Documents épistolaires concernant sa vie et ses oeuvres, Amberes, 1887-1909, vol. VI, p. 312]. Por enero de 1641 ya ha llegado a París [Abecedario de J. P. Mariette..., París, 1851-1860, p. 371]. Sabemos que de vuelta a Londres la enfermedad le impedía terminar los retratos de la princesa Ana y del príncipe de Oranje, y de su paso por Holanda para entregar las pinturas en persona [BARNES et al., op. cit. 2004, n. ${ }^{\circ}$ IV.163 y IV.164; véase la cronología de Van Dyck en p. 11].
} 
maestro $^{69}$, como viene siendo el caso con Peeter Thijs, que ha despertado el interés de la crítica en fechas recientes ${ }^{70}$.

Fecha de recepción: 12-II-2008

Fecha de aceptación: 19-V-2008

${ }^{69}$ Ya a principios del siglo XX se señalaba que sus retratos deben buscarse entre los atribuidos a Van Dyck [WuRZBACH, A., in Niederländischen Kunstler Lexikon, Viena-Leipzig, 1904-1911, II, p. 146].

${ }^{70}$ Véase lo citado en las notas 16, 39 y 40, así como: BALIS, A., "Pieter Thijs, Pepijn en een derde pseudo-Boeckhorst", en Jan Boeckhorst Medewerker van Rubens, Cat. Exp. Amberes, Rubenshuis, 1990, pp. 98-108; VLIEGHE, H., "Een wazige kijk op Erasmus II Quellin. Bedenkingen bij recent onderzoek", Jaarboek van het Koninklijk Museum voor Schone Kunsten, Antwerpen, 1993, p. 305; BALIS, A., "Van Dyck: Some Problems of Attributions", en Van Dyck 350, op. cit. 1994, p. 180; MAUfort, D., "Een zelfportret van Peeter Thijs de Oude (1624-1677) in de Koninklijke Musea voor Schone Kunsten van België", Bulletin des Musées Royaux des Beaux Arts de Belgique, 1994/1-4-1995/1-5, Bruselas, 2000, pp. 103-111; DíAz PADRÓN, M., "Un retrato de monje agustino con atribución a Van Dyck, restituido a Peter Thijs", Goya, n. ${ }^{\circ}$ 307-308, 2005, pp. 261-264; SAnzsalazar, J., "Una nueva pintura de Peter Thijs identificada en la Colegiata de Santa Gertrudis de Nivelles", Archivo Español de Arte, t. 82, n. ${ }^{\circ} 325,2009$, pp. 79-86]. 